\title{
NONCONVEX RETRACTS AND COMPUTATION FOR FIXED POINT INDEX IN CONES
}

\author{
Guowei Zhang — Pengcheng Li
}

\begin{abstract}
In this paper we construct two retracts in a cone by nonnegative functionals of convex and concave types, and an example is given to illustrate that the retracts are nonconvex. Then the nonconvex retracts are used to compute the fixed point index for the completely continuous operator in the domains $D_{1} \cap D_{2}$ and $D_{1} \cup D_{2}$, where $D_{1}$ and $D_{2}$ are bounded open sets in the cone. The computation for fixed point index can be applied to the existence and the more precise location of positive fixed points.
\end{abstract}

\section{Introduction}

Let $E$ be a real Banach space with the zero element denoted by $\theta$. A nonempty convex closed set $P \subset E$ is called a cone if it satisfies the following two conditions: (i) $\lambda x \in P$ for $x \in P$ and $\lambda \geq 0$; (ii) $\pm x \in P$ implies $x=\theta$. For the theory and properties of cone and fixed point index in Banach spaces we refer to [13], [14]. A nonnegative functional $\gamma: P \rightarrow[0,+\infty)$ is said to be convex or concave if $\gamma(t x+(1-t) y) \leq t \gamma(x)+(1-t) \gamma(y)$ and $\gamma(t x+(1-t) y) \geq$ $t \gamma(x)+(1-t) \gamma(y)$ for all $x, y \in P$ and $t \in[0,1]$, respectively. $\gamma$ is bounded if its range of bounded set in $E$ is bounded. For $D \subset P, \bar{D}$ and $\partial D$ are respectively the closure and boundary of $D$ in $P$. The open ball centered at $\theta$ with the radius

2010 Mathematics Subject Classification. Primary 47H11; Secondary 47H10.

Key words and phrases. Nonconvex retract, fixed point index, completely continuous operator. 
$R>0$ is denoted by $B_{R}=\{x \in E \mid\|x\|<R\}$ and $[x]$ stands for $x /\|x\|$ for $x \in E \backslash\{\theta\}$. Throughout this paper, the notations

$$
\begin{array}{ll}
D_{1}=\left\{x \in P \mid \alpha(x)<R_{1}\right\}, & D_{2}=\left\{x \in P \mid \beta(x)<R_{2}\right\}, \\
D_{1}^{\prime}=\left\{x \in P \mid \alpha(x)>R_{1}\right\}, & D_{2}^{\prime}=\left\{x \in P \mid \beta(x)>R_{2}\right\}
\end{array}
$$

are always used for the functionals $\alpha, \beta: P \rightarrow[0,+\infty)$ and the constants $R_{1}, R_{2}>0$.

Computation for fixed point index in cones and topological degrees about nonlinear completely continuous operators plays a very important role in the fixed point theory, see the references [1]-[10], [12]-[23] listed in this paper and others. Krasnosel'skii is the first author who considered fixed point index results about cone compression/expansion (see [15]). In [1], [3] there are the following results of computation for fixed point index.

Theorem 1.1. Let $P$ be a cone in $E, \alpha, \beta: P \rightarrow[0,+\infty)$ be continuous functionals, $D_{1}$ and $D_{2}$ be nonempty bounded subsets of $P$.

(a) Suppose that $A: \bar{D}_{1} \rightarrow P$ is a completely continuous operator with

$$
\inf _{x \in \partial D_{1}}\|A x\|>0 \text { and } A\left(\partial D_{1}\right) \subset \bar{D}_{1}^{\prime} .
$$

If $\alpha(\lambda x) \leq \lambda \alpha(x)$ for $x \in \partial D_{1}, \lambda \in(0,1]$, then the fixed point index $i\left(A, D_{1}, P\right)=0$

(b) Suppose that $A: \bar{D}_{2} \rightarrow P$ is a completely continuous operator with $A\left(\partial D_{2}\right) \subset$ $\bar{D}_{2}$. If $\beta(\theta)=0$ and $\beta(\mu x) \geq \mu \beta(x)$ for $x \in \partial D_{2}, \mu \geq 1$, then the fixed point index $i\left(A, D_{2}, P\right)=1$.

Theorem 1.2. Let $P$ be a cone in $E, \alpha: P \rightarrow[0,+\infty)$ be a continuous convex functional and $\beta: P \rightarrow[0,+\infty)$ be a continuous concave functional, $D_{1}$ and $D_{2}$ be nonempty bounded subsets of $P$ with $D_{1} \cap D_{2}^{\prime} \neq \emptyset$. Suppose that $A: P \rightarrow P$ is a completely continuous operator.

(a) If $A x \in D_{1}$ for $x \in\left(\partial D_{1}\right) \cap \bar{D}_{2}^{\prime}$ and $x \in\left(\partial D_{1}\right) \cap\left\{x \in P \mid A x \in D_{2}\right\}$, then the fixed point index $i\left(A, D_{1}, P\right)=1$.

(b) If $A x \in D_{2}^{\prime}$ for $x \in\left(\partial D_{2}\right) \cap \bar{D}_{1}$ and $x \in\left(\partial D_{2}\right) \cap\left\{x \in P \mid A x \in D_{1}^{\prime}\right\}$, then the fixed point index $i\left(A, D_{2}, P\right)=0$.

Through these computations for fixed point index, the fixed point theorems of expansion-compression type in cones were deduced and applied to the existence and the location of positive solutions for differential and difference equations. In this paper we shall discuss the fixed point index in the domains $D_{1} \cap D_{2}$ and $D_{1} \cup D_{2}$ which can be applied to the existence and the more precise location of positive fixed points, and thus positive solutions for differential and difference equations under different conditions from those in previous work. 
First we construct two nonconvex retracts in a cone by nonnegative functionals of convex and concave types, and then the retracts are used to compute the fixed point index for the completely continuous operator in the domains $D_{1} \cap D_{2}$ and $D_{1} \cup D_{2}$. A subset $X \subset E$ is called a retract of $E$ if there exists a continuous mapping $r: E \rightarrow X$, a retraction, satisfying $r(x)=x, x \in X$. By a theorem due to Dugundji [11], every nonempty closed convex subset of $E$ is a retract of $E$. An example is given to illustrate that the retracts obtained in this paper are nonconvex.

\section{Nonconvex retracts}

Theorem 2.1. Let $P$ be a cone in $E, \alpha: P \rightarrow[0,+\infty)$ be a continuous convex functional and $\beta: P \rightarrow[0,+\infty)$ be a bounded continuous concave functional with $\alpha(\theta)=\beta(\theta)=0$ and $\alpha(x)>0, \beta(x)>0$ for $x \neq \theta$, both $\{x \in P \mid \alpha(x) \leq R\}$ and $\{x \in P \mid \beta(x) \leq R\}$ be bounded for all $R>0$. If

$$
\beta(\mu x)>\beta(x), \quad \text { for all } \mu>1, x \in P \backslash\{\theta\},
$$

then $\bar{D}_{1} \cap \bar{D}_{2}$ is a retract of $E$.

Proof. Since both $\alpha$ and $\beta$ are continuous with $\alpha(\theta)=\beta(\theta)=0$, there exist nonzero elements in $\bar{D}_{1} \cap \bar{D}_{2}$, i.e. $\bar{D}_{1} \cap \bar{D}_{2} \neq \emptyset$.

(a) Since $\bar{D}_{1}$ is a closed convex set, there exists a retraction $g_{1}: E \rightarrow \bar{D}_{1}$.

(b) If $\bar{D}_{1} \cap \bar{D}_{2}^{\prime}=\emptyset$, then $\bar{D}_{1} \subset \bar{D}_{2}$ and $\bar{D}_{1} \cap \bar{D}_{2}=\bar{D}_{1}$ is a retract of $E$. Afterwards we may suppose that $\bar{D}_{1} \cap \bar{D}_{2}^{\prime} \neq \emptyset$. Since $\bar{D}_{1}$ is bounded, there exists a constant $R_{1}^{\prime}>0$ such that $\|x\| \leq R_{1}^{\prime}$, for all $x \in \bar{D}_{1} \cap \bar{D}_{2}^{\prime}$. Because $\beta(x)$ is a bounded functional, we have a constant $M>R_{2}$ such that $\beta(x) \leq M$, for all $x \in \bar{D}_{1} \cap \bar{D}_{2}^{\prime}$. Due to the boundedness of $\{x \in P \mid \beta(x) \leq M+1\}$, we know that there exists a constant $R_{2}^{\prime}>R_{1}^{\prime}$ such that $\beta(x)>M+1$ for $x \in P \cap \partial B_{R_{2}^{\prime}}$. Owing to $\theta \notin \bar{D}_{1} \cap \bar{D}_{2}^{\prime}$, we can define

$$
g_{2}(x)=\frac{\beta\left(R_{2}^{\prime}[x]\right)-R_{2}}{\beta\left(R_{2}^{\prime}[x]\right)-\beta(x)}\left(x-R_{2}^{\prime}[x]\right), \quad \text { for all } x \in \bar{D}_{1} \cap \bar{D}_{2}^{\prime} .
$$

Obviously, $g_{2}$ is continuous.

(c) For $x \in \bar{D}_{1} \cap \bar{D}_{2}^{\prime}$, define

$$
g_{3}(x)= \begin{cases}g_{2}(x)+R_{2}^{\prime}[x], & \left\|g_{2}(x)\right\| \leq R_{2}^{\prime} \\ \theta, & \left\|g_{2}(x)\right\|>R_{2}^{\prime}\end{cases}
$$

Thus $g_{3}: \bar{D}_{1} \cap \bar{D}_{2}^{\prime} \rightarrow P$ is continuous. In fact, if $\left\|g_{2}(x)\right\| \leq R_{2}^{\prime}$, i.e.

$$
\left\|\frac{\beta\left(R_{2}^{\prime}[x]\right)-R_{2}}{\beta\left(R_{2}^{\prime}[x]\right)-\beta(x)}\left(x-R_{2}^{\prime}[x]\right)\right\|=\frac{\beta\left(R_{2}^{\prime}[x]\right)-R_{2}}{\beta\left(R_{2}^{\prime}[x]\right)-\beta(x)}\left(R_{2}^{\prime}-\|x\|\right) \leq R_{2}^{\prime},
$$


then

$$
g_{3}(x)=\left(\frac{\beta\left(R_{2}^{\prime}[x]\right)-R_{2}}{\beta\left(R_{2}^{\prime}[x]\right)-\beta(x)}\left(\|x\|-R_{2}^{\prime}\right)+R_{2}^{\prime}\right)[x] \in P,
$$

which implies that $g_{3}: \bar{D}_{1} \cap \bar{D}_{2}^{\prime} \rightarrow P$. When $\left\|g_{2}(x)\right\|=R_{2}^{\prime}$, by $(2.2)$ we have $g_{3}(x)=\theta$ and hence $g_{3}(x)$ is continuous.

(d) Define

$$
g_{4}(x)= \begin{cases}g_{3}(x), & x \in \bar{D}_{1} \cap \bar{D}_{2}^{\prime} \\ x, & x \in \bar{D}_{1} \cap \bar{D}_{2} .\end{cases}
$$

For $x \in\left\{x \in P \mid \beta(x)=R_{2}, \alpha(x) \leq R_{1}\right\}$, we have that $g_{2}(x)=x-R_{2}^{\prime}[x]$ and $\left\|g_{2}(x)\right\|=R_{2}^{\prime}-\|x\|<R_{2}^{\prime}$. Therefore $g_{3}(x)=x$ and $g_{4}: D_{1} \rightarrow P$ is well defined and continuous.

(e) In the following we shall show that $\beta\left(g_{3}(x)\right) \leq R_{2}, \alpha\left(g_{3}(x)\right) \leq R_{1}$ for $x \in \bar{D}_{1} \cap \bar{D}_{2}^{\prime}$, that is, $g_{4}: D_{1} \rightarrow \bar{D}_{1} \cap \bar{D}_{2}$.

Actually when $\left\|g_{2}(x)\right\|>R_{2}^{\prime}$, we have that $g_{3}(x)=\theta$, and hence $\alpha\left(g_{3}(x)\right)=$ $0 \leq R_{1}, \beta\left(g_{3}(x)\right)=0 \leq R_{2}$.

When $\left\|g_{2}(x)\right\| \leq R_{2}^{\prime}$, it follows from $\beta(x) \geq R_{2}$ that

$$
\frac{\beta\left(R_{2}^{\prime}[x]\right)-R_{2}}{\beta\left(R_{2}^{\prime}[x]\right)-\beta(x)} \geq 1
$$

and

$$
\begin{aligned}
g_{3}(x) & =\frac{\beta\left(R_{2}^{\prime}[x]\right)-R_{2}}{\beta\left(R_{2}^{\prime}[x]\right)-\beta(x)}\left(x-R_{2}^{\prime}[x]\right)+R_{2}^{\prime}[x], \\
x & =\frac{\beta\left(R_{2}^{\prime}[x]\right)-\beta(x)}{\beta\left(R_{2}^{\prime}[x]\right)-R_{2}} g_{3}(x)+\left(1-\frac{\beta\left(R_{2}^{\prime}[x]\right)-\beta(x)}{\beta\left(R_{2}^{\prime}[x]\right)-R_{2}}\right) R_{2}^{\prime}[x] .
\end{aligned}
$$

By the concavity of $\beta$, we have

$$
\begin{gathered}
\beta(x) \geq \frac{\beta\left(R_{2}^{\prime}[x]\right)-\beta(x)}{\beta\left(R_{2}^{\prime}[x]\right)-R_{2}} \beta\left(g_{3}(x)\right)+\left(1-\frac{\beta\left(R_{2}^{\prime}[x]\right)-\beta(x)}{\beta\left(R_{2}^{\prime}[x]\right)-R_{2}}\right) \beta\left(R_{2}^{\prime}[x]\right), \\
(2.3) \beta\left(g_{3}(x)\right) \leq \frac{\beta\left(R_{2}^{\prime}[x]\right)-R_{2}}{\beta\left(R_{2}^{\prime}[x]\right)-\beta(x)} \beta(x)-\left(\frac{\beta\left(R_{2}^{\prime}[x]\right)-R_{2}}{\beta\left(R_{2}^{\prime}[x]\right)-\beta(x)}-1\right) \beta\left(R_{2}^{\prime}[x]\right)=R_{2} .
\end{gathered}
$$

On the other hand, when $\left\|g_{2}(x)\right\| \leq R_{2}^{\prime}$, if

$$
\frac{\beta\left(R_{2}^{\prime}[x]\right)-R_{2}}{\beta\left(R_{2}^{\prime}[x]\right)-\beta(x)}\left(1-R_{2}^{\prime} \frac{1}{\|x\|}\right)+R_{2}^{\prime} \frac{1}{\|x\|}>1
$$

from (2.1) we have

$$
\beta\left(g_{3}(x)\right)=\beta\left(\left(\frac{\beta\left(R_{2}^{\prime}[x]\right)-R_{2}}{\beta\left(R_{2}^{\prime}[x]\right)-\beta(x)}\left(1-R_{2}^{\prime} \frac{1}{\|x\|}\right)+R_{2}^{\prime} \frac{1}{\|x\|}\right) x\right)>\beta(x) .
$$

Since $\beta(x) \geq R_{2}$, it follows that $\beta\left(g_{3}(x)\right)>R_{2}$, which contradicts $(2.3)$, and thus

$$
0 \leq \frac{\beta\left(R_{2}^{\prime}[x]\right)-R_{2}}{\beta\left(R_{2}^{\prime}[x]\right)-\beta(x)}\left(1-R_{2}^{\prime} \frac{1}{\|x\|}\right)+R_{2}^{\prime} \frac{1}{\|x\|} \leq 1 .
$$


By the convexity of $\alpha$,

$$
\begin{aligned}
\alpha\left(g_{3}(x)\right) & =\alpha\left(\left(\frac{\beta\left(R_{2}^{\prime}[x]\right)-R_{2}}{\beta\left(R_{2}^{\prime}[x]\right)-\beta(x)}\left(1-R_{2}^{\prime} \frac{1}{\|x\|}\right)+R_{2}^{\prime} \frac{1}{\|x\|}\right) x\right) \\
& \leq\left(\frac{\beta\left(R_{2}^{\prime}[x]\right)-R_{2}}{\beta\left(R_{2}^{\prime}[x]\right)-\beta(x)}\left(1-R_{2}^{\prime} \frac{1}{\|x\|}\right)+R_{2}^{\prime} \frac{1}{\|x\|}\right) \alpha(x) \leq \alpha(x) \leq R_{1} .
\end{aligned}
$$

(f) Let $g(x)=g_{4}\left(g_{1}(x)\right)$, for all $x \in E$, then $g: E \rightarrow \bar{D}_{1} \cap \bar{D}_{2}$ is a retraction. $\square$

Theorem 2.2. Let $P$ be a cone in $E, \alpha: P \rightarrow[0,+\infty)$ be a continuous functional and $\beta: P \rightarrow[0,+\infty)$ be a continuous concave functional with $\alpha(\theta)=$ $\beta(\theta)=0$ and $\alpha(x)>0, \beta(x)>0$ for $x \neq \theta$. If $D_{1}^{\prime} \cap D_{2}^{\prime} \neq \emptyset,(2.1)$ holds and

$$
\alpha(\lambda x) \leq \lambda \alpha(x), \quad \text { for all } \lambda \in[0,1], x \in P,
$$

then $\bar{D}_{1}^{\prime} \cap \bar{D}_{2}^{\prime}$ is a retract of $E$.

Proof. (a) Since $\bar{D}_{2}^{\prime}$ is a closed convex set, there exists a retraction

$$
g_{1}: E \rightarrow \bar{D}_{2}^{\prime} \text {. }
$$

(b) If $\bar{D}_{1} \cap \bar{D}_{2}^{\prime}=\emptyset$, then $\bar{D}_{1}^{\prime} \supset \bar{D}_{2}^{\prime}$ and $\bar{D}_{1}^{\prime} \cap \bar{D}_{2}^{\prime}=\bar{D}_{2}^{\prime}$ is a retract of $E$. Afterwards we may suppose that $\bar{D}_{1} \cap \bar{D}_{2}^{\prime} \neq \emptyset$. Owing to $\theta \notin \bar{D}_{1} \cap \bar{D}_{2}^{\prime}$, we can define

$$
g_{2}(x)= \begin{cases}\frac{R_{1}}{\alpha(x)} x, & x \in \bar{D}_{1} \cap \bar{D}_{2}^{\prime} ; \\ x, & x \in \bar{D}_{1}^{\prime} \cap \bar{D}_{2}^{\prime} .\end{cases}
$$

Because $g_{2}(x)=x$ if $\alpha(x)=R_{1}$ and $\beta(x) \geq R_{2}$, we have that $g_{2}(x)$ is continuous on $\bar{D}_{2}^{\prime}$.

(c) For $x \in \bar{D}_{1} \cap \bar{D}_{2}^{\prime}, g_{2}(x)=R_{1} x / \alpha(x)$, i.e. $x=\alpha(x) g_{2}(x) / R_{1}$. It follows from $\alpha(x) \leq R_{1}$ that $\alpha(x) / R_{1} \leq 1$, and thus by (2.4),

$$
\alpha(x)=\alpha\left(\frac{\alpha(x)}{R_{1}} g_{2}(x)\right) \leq \frac{\alpha(x)}{R_{1}} \alpha\left(g_{2}(x)\right),
$$

that is, $\alpha\left(g_{2}(x)\right) \geq R_{1}$. We have from (2.1) that

$$
\beta\left(g_{2}(x)\right)=\beta\left(\frac{R_{1}}{\alpha(x)} x\right) \geq \beta(x) \geq R_{2},
$$

and then $g_{2}: \bar{D}_{2}^{\prime} \rightarrow \bar{D}_{1}^{\prime} \cap \bar{D}_{2}^{\prime}$.

(d) Let $g(x)=g_{2}\left(g_{1}(x)\right)$, for all $x \in E$, then $g: E \rightarrow \bar{D}_{1}^{\prime} \cap \bar{D}_{2}^{\prime}$ is a retraction.

\section{Computation for the fixed point index}

In this section we shall use the retracts obtained above to compute the fixed point index for nonlinear completely continuous operators. The next theorem follows the idea from [19]. 
TheOREm 3.1. Let $P$ be a cone in $E$ and $\Omega$ be a bounded open set in $P$, $A: \bar{\Omega} \rightarrow P$ be completely continuous with $A x \neq x$, for all $x \in \partial \Omega$. Suppose that $D \subset P$ is a retract of $E$ satisfying $A(\partial \Omega) \subset D$.

(a) If $D \subset \bar{\Omega}$, then the fixed point index $i(A, \Omega, P)=1$;

(b) If $D \cap \Omega=\emptyset$, then the fixed point index $i(A, \Omega, P)=0$.

Proof. Let $g: E \rightarrow D$ be a retraction.

(a) Take $R$ sufficiently large such that $\bar{\Omega} \subset P_{R}=\{x \in P \mid\|x\|<R\}$. By the extension theorem, $\left.A\right|_{\partial \Omega}$ has a completely continuous extension $A_{1}: \bar{P}_{R} \rightarrow P$ with $A_{1} x=A x$ for $x \in \partial \Omega$. Denote $A_{2}=g A_{1}$, then $A_{2}: \bar{P}_{R} \rightarrow D$ is completely continuous and $A_{2} x=A x$ for $x \in \partial \Omega$ since $A(\partial \Omega) \subset D$. It follows from $D \subset$ $\bar{\Omega} \subset P_{R}$ and the homotopy invariance of fixed point index that

$$
i\left(A_{2}, P_{R}, P\right)=i\left(\theta, P_{R}, P\right)=1 .
$$

Notice that $A_{2}: \bar{P}_{R} \rightarrow D \subset \bar{\Omega}$ and $A x \neq x$ for $x \in \partial \Omega$, then $A_{2}$ has no fixed point in $\bar{P}_{R} \backslash \Omega$ and hence

$$
i\left(A_{2}, P_{R}, P\right)=i\left(A_{2}, \Omega, P\right)=i(A, \Omega, P) .
$$

Therefore, from (3.1) and (3.2) it follows that $i(A, \Omega, P)=1$.

(b) $\left.A\right|_{\partial \Omega}$ has a completely continuous extension $A_{3}: \bar{\Omega} \rightarrow P$ with $A_{3} x=A x$ for $x \in \partial \Omega$. Denote $A_{4}=g A_{3}$, thus $A_{4}: \bar{\Omega} \rightarrow D$ is completely continuous and $A_{4} x=A x$ for $x \in \partial \Omega$ since $A(\partial \Omega) \subset D$. If $i(A, \Omega, P) \neq 0$, then $i\left(A_{4}, \Omega, P\right) \neq 0$ and $A_{4}$ has a fixed point in $D \cap \Omega$, which contradicts $D \cap \Omega=\emptyset$.

Theorem 3.2. Let $P$ be a cone in $E, \alpha: P \rightarrow[0,+\infty)$ be a continuous convex functional and $\beta: P \rightarrow[0,+\infty)$ be a bounded continuous concave functional with $\alpha(\theta)=\beta(\theta)=0$ and $\alpha(x)>0, \beta(x)>0$ for $x \neq \theta$, both $\{x \in P \mid \alpha(x) \leq R\}$ and $\{x \in P \mid \beta(x) \leq R\}$ be bounded for all $R>0, A: P \rightarrow P$ be completely continuous. Suppose that (2.1) holds.

(a) If $A\left(\partial\left(D_{1} \cap D_{2}\right)\right) \subset \bar{D}_{1} \cap \bar{D}_{2}$ with $A x \neq x$, for all $x \in \partial\left(D_{1} \cap D_{2}\right)$, then $i\left(A, D_{1} \cap D_{2}, P\right)=1$;

(b) If $A\left(\partial\left(D_{1} \cap D_{2}\right)\right) \subset \bar{D}_{1}^{\prime} \cap \bar{D}_{2}^{\prime}$ with $A x \neq x$, for all $x \in \partial\left(D_{1} \cap D_{2}\right)$, then $i\left(A, D_{1} \cap D_{2}, P\right)=0$

(c) If $A\left(\partial\left(D_{1} \cup D_{2}\right)\right) \subset \bar{D}_{1} \cap \bar{D}_{2}$ with $A x \neq x$, for all $x \in \partial\left(D_{1} \cup D_{2}\right)$, then $i\left(A, D_{1} \cup D_{2}, P\right)=1$;

(d) If $A\left(\partial\left(D_{1} \cup D_{2}\right)\right) \subset \bar{D}_{1}^{\prime} \cap \bar{D}_{2}^{\prime}$ with $A x \neq x$, for all $x \in \partial\left(D_{1} \cup D_{2}\right)$, then $i\left(A, D_{1} \cup D_{2}, P\right)=0$.

Proof. It is clear that $\bar{D}_{1}^{\prime} \cap \bar{D}_{2}^{\prime} \neq \emptyset$ since both $\{x \in P \mid \alpha(x) \leq R\}$ and $\{x \in P \mid \beta(x) \leq R\}$ are bounded for all $R>0$. By the convexity of $\alpha$ and $\alpha(\theta)=0$ we know that (2.4) is satisfied. From Theorems 2.1 and 2.2 it follows 
that $\bar{D}_{1} \cap \bar{D}_{2}$ and $\bar{D}_{1}^{\prime} \cap \bar{D}_{2}^{\prime}$ are retracts of $E$, and then Theorem 3.1 tells us that the conclusions of this theorem holds.

Corollary 3.3. Under the same conditions as those in Theorem 3.2, if $\left(\bar{D}_{1} \cup \bar{D}_{2}\right) \backslash\left(D_{1} \cap D_{2}\right) \neq \emptyset$ and

(a) $A\left(\partial\left(D_{1} \cap D_{2}\right)\right) \subset \bar{D}_{1} \cap \bar{D}_{2}, A\left(\partial\left(D_{1} \cup D_{2}\right)\right) \subset \bar{D}_{1}^{\prime} \cap \bar{D}_{2}^{\prime}$; or

(b) $A\left(\partial\left(D_{1} \cap D_{2}\right)\right) \subset \bar{D}_{1}^{\prime} \cap \bar{D}_{2}^{\prime}, A\left(\partial\left(D_{1} \cup D_{2}\right)\right) \subset \bar{D}_{1} \cap \bar{D}_{2}$,

then $A$ has a fixed point in $\left(\bar{D}_{1} \cup \bar{D}_{2}\right) \backslash\left(D_{1} \cap D_{2}\right)$.

\section{An example}

In this section we will give an example to illustrate that the retracts obtained are nonconvex.

Let $E=C[0,1]$ be Banach space with the norm $\|x\|=\max _{0 \leq t \leq 1}|x(t)|$, for all $x \in C[0,1]$,

$$
P=\left\{x \in C[0,1]|| x(t) \geq 0, \text { for all } t \in[0,1], \min _{t \in[1 / 3,2 / 3]} x(t) \geq \frac{1}{9}\|x\|\right\} .
$$

Obviously, $P$ is a cone in $E$. Define

$$
\alpha(x)=\max _{t \in[1 / 3,2 / 3]} x(t), \beta(x)=\min _{t \in[1 / 3,2 / 3]} x(t)
$$

for $x \in P$, it is clear that $\alpha: P \rightarrow[0,+\infty)$ is a continuous convex functional and $\beta: P \rightarrow[0,+\infty)$ is a bounded continuous concave functional with $\alpha(\theta)=$ $\beta(\theta)=0$ and $\alpha(x)>0, \beta(x)>0$ for $x \neq \theta$, both $\{x \in P \mid \alpha(x) \leq R\}$ and $\{x \in P \mid \beta(x) \leq R\}$ are bounded for all $R>0$. Moreover, (2.1) and (2.4) are satisfied.

Let $R_{1}=7 / 9$ and $R_{2}=5 / 18$. If we take $x_{1}(t)=5 t / 6$ and $x_{2}(t)=5(1-t) / 6$ for $t \in[0,1]$, then $x_{1}, x_{2} \in \bar{D}_{1} \cap \bar{D}_{2}$. However $\alpha\left(\left(x_{1}+x_{2}\right) / 2\right)=5 / 12>R_{2}$, thus $\bar{D}_{1} \cap \bar{D}_{2}$ is not convex. If we take $x_{3}(t)=7 t / 6$ and $x_{4}(t)=7(1-t) / 6$ for $t \in[0,1]$, then $x_{3}, x_{4} \in \bar{D}_{1}^{\prime} \cap \bar{D}_{2}^{\prime}$. However $\alpha\left(\left(x_{3}+x_{4}\right) / 2\right)=7 / 12<R_{1}$, thus $\bar{D}_{1}^{\prime} \cap \bar{D}_{2}^{\prime}$ is not convex. Besides, if we take $x_{5}(t)=t$ and $x_{6}(t)=2(t-1)^{2}$ for $t \in[0,1]$, then it is easy to see that $x_{5} \in \bar{D}_{1} \backslash D_{2}$ and $x_{6} \in \bar{D}_{2} \backslash D_{1}$, i.e. $\left(\bar{D}_{1} \cup \bar{D}_{2}\right) \backslash\left(D_{1} \cap D_{2}\right) \neq \emptyset$.

\section{REFERENCES}

[1] R.I. Avery and D.R. Anderson, Fixed point theorem of cone expansion and compression of functional type, J. Difference Equ. Appl. 8 (2002), 1073-1083.

[2] R.P. Agarwal, R.I. Avery, J. Henderson and D. O'Regan, The five functionals fixed point theorem generalized to multivalued maps, J. Nonlinear Convex Anal. 4 (2003), 455-462.

[3] D.R. Anderson, R.I. Avery and J. Henderson, Some fixed point theorem of LeggettWilliams type, Rocky Mountain J. Math. 41 (2011), 371-386. 
[4] Functional expansion-compression fixed point theorem of Leggett-Williams type, Electron J. Differential Equations 63 (2010), 1-9.

[5] R.I. AVEry AND J. Henderson, Two positive fixed points of nonlinear operators on ordered Banach spaces, Comm. Appl. Nonlinear Anal. 8 (2001), 27-36.

[6] _ An extension of the five functionals fixed point theorem, J. Difference Equ. Appl. 1 (2000), 275-290.

[7] R.I. Avery, J. Henderson and D. O'Regan, Functional compression-expansion fixed point theorem, Electron J. Differential Equations 22 (2008), 1-12.

[8] A dual of the compression-expansion fixed point theorems, Fixed Point Theory Appl. (2007), Article ID 90715, 11 pages.

[9] R.P. Agarwal And D. O'Regan, A generalization of the Petryshyn-Leggett-Williams fixed point theorem with applications to integral inclusions, Appl. Math. Comput. 123 (2001), 263-274.

[10] Y. CUI, F. WANG AND Y. ZOU, Computation for the fixed point index and its applications, Nonlinear Anal. 71 (2009), 219-226.

[11] J. Dugundj, An extension of Tietze theorem, Pacific J. Math. 1 (1951), 353-367.

[12] M. Feng, X. Zhang And W. GE, Positive fixed point of strict set contraction operators on ordered Banach spaces and applications, Abstr. Appl. Anal. (2010), Article ID 439137, 13 pages.

[13] A. Granas and J. Dugundji, Fixed Point Theory, Springer-Verlag, New York, 2003.

[14] D. Guo and V. Lakshmikantham, Nonlinear Problems in Abstract Cones, Academic Press, San Diego, 1988.

[15] M.A. Krasnosel'skǏ, Positive Solutions of Operator Equations, P. Noordhoff, Gröningen, 1964 .

[16] F. Li AND G. Han, Generalization for Amann's and Leggett-Williams' three-solution theorems and applications, J. Math. Anal. Appl. 298 (2004), 638-654.

[17] R.W. LEgGett And L.R. Williams, Multiple positive fixed points of nonlinear operators on ordered Banach spaces, Indiana Univ. Math. J. 28 (1979), 673-688.

[18] D. O'Regan And R. PReCup, Compression-expansion fixed point theorem in two norms and applications, J. Math. Anal. Appl. 309 (2005), 383-391.

[19] J. Sun, Nonlinear Functional Analysis and Its Applications, Science Press, Beijing, 2008. (Chinese)

[20] D. Sun And G. Zhang, Computing the topological degrees via semi-concave functionals, Topol. Methods Nonlinear Anal. 39 (2012), 107-117.

[21] D. Sun, G. Zhang AND T. ZhAng, Fixed point theorem about cone expansion and compression of concave functional type, Acta Math. Sinica 53 (2010), 847-852. (Chinese)

[22] G. Zhang AND J. Sun, A generalization of cone expansion and compression fixed point theorem and applications, Nonlinear Anal. 67 (2007), 579-586.

[23] G. Zhang, J. Sun And T. Zhang, Fixed point theorem of domain compression and expansion of convex functional type, Acta Math. Sinica 51 (2008), 517-522. (Chinese)

Guowei Zhang And Pengcheng Li

Department of Mathematics

Northeastern University

Shenyang 110819, P.R.CHINA

E-mail address: gwzhang@mail.neu.edu.cn

TMNA : Volume $43-2014-\mathrm{N}^{\circ} 2$ 